\title{
A LARGE PISTON DISPLACEMENT MEMS MIRROR WITH ELECTROTHERMAL LADDER ACTUATOR ARRAYS FOR FOURIER TRANSFORM SPECTROSCOPY APPLICATIONS

\author{
S.R. Samuelson ${ }^{*}$ and H. Xie
}

Department of Electrical and Computer Engineering, University of Florida, Gainesville, Florida, USA

\begin{abstract}
A large displacement piston motion micromirror is designed, fabricated and tested with device features tuned to application in a hand-portable Fourier Transform Spectroscopy (FTS) system. The fabricated MEMS mirror is based on electrothermal actuation and has a footprint of $1.9 \times 1.9 \times 0.5 \mathrm{~mm}^{3}$ with a mirror aperture of $1.02 \mathrm{~mm}$. The application optimized device holds key features of ultralow maximum tilt of $0.25^{\circ}$, a large, strongly linear motion of $90 \mu \mathrm{m}$ achievable at only $1.2 \mathrm{~V} \mathrm{dc}$ and a maximum theoretical spectral resolution of $55.6 \mathrm{~cm}^{-1}$. This device is further characterized with pertinent characteristics for applicability to rapid scan FTS system configurations.
\end{abstract}

\section{INTRODUCTION}

MEMS-based FTS systems based on amplitude division interferometry possess the same benefits as their macro-scale counterparts, namely the multiplex and throughput advantages, which result in fast measurement and high sensitivity [1]. These systems are constrained by spectral resolution, which is limited by the achievable optical path difference (OPD). These systems in the micro-scale have been investigated and demonstrated both based on fabrication via LIGA and surface/bulk micromachining [2]. The primary actuation methodologies employed include electrostatic, electrothermal, and electromagnetic. An electrostatic device that includes a fully integrated system can achieve a maximum displacement of $25 \mu \mathrm{m}$ [3] while a fabricated electrostatic mirror as a standalone device has been reported to achieve up to $38.5 \mu \mathrm{m}$ displacement via in plane actuation [4]. Another device is also a discrete mirror that stacks comb drives to achieve an out of plane displacement of $200 \mu \mathrm{m}$ at resonance under vacuum at $100 \mathrm{~Pa}$ [5]. Electromagnetic devices have been constructed via LIGA technology and are able to achieve $54 \mu \mathrm{m}$ effective displacement [6].

Prior work has been accomplished with electrothermal devices that have yielded a device with $308 \mu \mathrm{m}$ of effective displacement [7]. The work employed an LSF-LVD electrothermal micromirror that used both sides of the mirror surface in a tilt insensitive FTS system to combat the high tilt inherent in the mirror design. This system allowed four times the OPD via the path length varying through both arms of the FTS system. This contrasts with the usual doubling of path length in traditional Michelson interferometer systems and thus allowed a spectral resolution of $8.1 \mathrm{~cm}^{-1}$. The shortcoming of this system stems from the all-purpose design orientation of the micromirror that allowed large displacement but additionally generated large tilt that necessitated the tilt insensitive system, which added additional optical components and thus increased system size. Additionally, tilt compensation only removed beam misalignment from the actuator axis but did not restrict travel of the matched beams in tandem or remove tilt for the off-actuator axis.

This work carries over the goals of the prior work to create a system that is compact, portable and of low cost such that it may be employed in areas such as chemical analysis and biohazard detection [7]. To add to these goals, it is of paramount importance to reduce tilt while not excessively sacrificing displacement as well as increasing robustness of the system for portable use.

\section{MEMS MIRROR DESIGN}

Actuator Structure and Function

With these goals firmly in mind, the basic conceptualization of the electrothermal actuator was chosen to be based on a foldeddual-S-shaped-bimorph (FDSB) electrothermal actuator design to achieve large displacement at low drive voltage [8]. The fundamental principle of electrothermal actuation is to induce a temperature change through Joule heating which is generated by applying an electrical current to a resistor built in the bimorph; as a consequence of this heating, the difference of thermal stresses due to different coefficients of thermal expansion (CTEs) between the two primary layers of the bimorph results in bending of the cantilevered structure. The core of this actuator design is the Sshaped bimorph (Fig. 1(a)), which consists of three sections $\mathrm{L}_{1}, \mathrm{~L}_{2}$ and $\mathrm{L}_{3}$ which contain the following thin film layers of $\mathrm{Al} / \mathrm{SiO}_{2}$, $\mathrm{SiO}_{2} / \mathrm{Al} / \mathrm{SiO}_{2}$ and $\mathrm{SiO}_{2} / \mathrm{Al}$ respectively. The initial position of this beam, after release from the silicon substrate on which it is fabricated, will demonstrate the S-shaped structure as shown in Fig. 1(b) which is given via FEM simulation in COMSOL [9]. Section $\mathrm{L}_{1}$ generates initial upward curvature while $\mathrm{L}_{3}$ generates downward curvature since $\mathrm{Al}$ has much larger CTE than $\mathrm{SiO}_{2}$. Additionally, section $\mathrm{L}_{2}$ provides an overlap that protects the junction between the two active sections and provides approximate self-compensation of thermal strain for an effectively straight section.

(a)

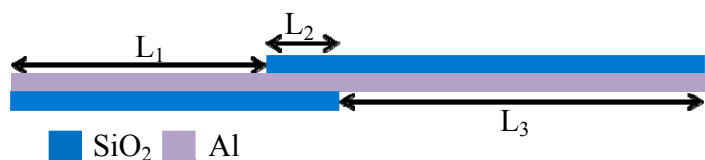

(b)

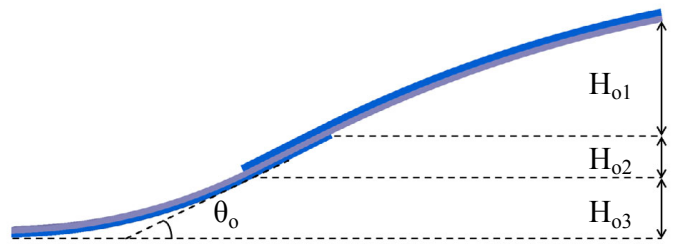

Figure 1: (a) Three constituent sections of S-shaped bimorph, (b) Initial vertical displacement on release.

A hinge is implemented that is of the same approximately self-compensated structure as $\mathrm{L}_{2}$ which joins two S-curves to form a FDSB actuator. This actuator structure results in a pure vertical displacement. In order to further increase the vertical displacement, two FDSB actuators are connected in series to form a ladder actuator (Fig. 2). With $\mathrm{R}_{\mathrm{o} 1}$ and $\mathrm{R}_{\mathrm{o} 3}$ signifying the initial radius of curvature of $\mathrm{L}_{1}$ and $\mathrm{L}_{3}$ respectively, the initial displacement of the FDSB ladder actuator can be expressed as follows (Eq. 1-3):

$$
\begin{aligned}
& H_{o}=4\left(H_{o 1}+H_{o 2}+H_{o 3}\right) \\
& H_{o}=4\left[R_{o 1}\left(1-\cos \theta_{o}\right)+L_{2} \sin \theta_{o}+R_{o 3}\left(1-\cos \theta_{o}\right)\right]
\end{aligned}
$$




$$
H_{o}=4\left[R_{o 1}\left(1-\cos \left(\frac{L_{1}}{R_{o 1}}\right)\right)+L_{2} \sin \left(\frac{L_{1}}{R_{o 1}}\right)+R_{o 3}\left(1-\cos \left(\frac{L_{1}}{R_{o 1}}\right)\right)\right](3)
$$

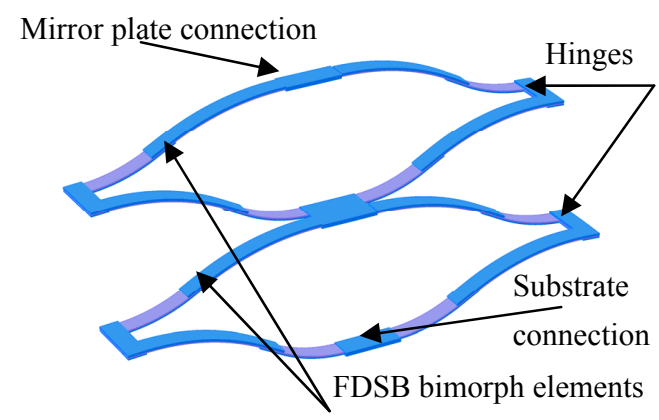

Figure 2: Full ladder actuator FEM simulation with stacked FDSB bimorph elements.

In addition to these equations which determine initial displacement of the actuator structure, an analytical model for the induced-strain actuator structure can determine the radii of curvature of the separate sections where thermal stress is the only contribution to the induced strain [10]. The primary components of the actuator are the bimorph layers of $\mathrm{SiO}_{2}$ and $\mathrm{Al}$. However, there are additional layers in the actuator that qualifies as a multimorph. These different layers include thin $\mathrm{SiO}_{2}$ and $\mathrm{Cr}$ layers that function as insulation and adhesion layers. Finally, there is a Pt layer that functions as a heater to allow greater Joule heating uniformity. Although these layers are contributory to the actuator structure and classify it as a multimorph as shown in accurate detail in Fig 3, the primary functional layers remain that of the bimorph and consideration of the full multimorph stack can be made in FEM simulation and experimental results to show this as a valid simplification [8].

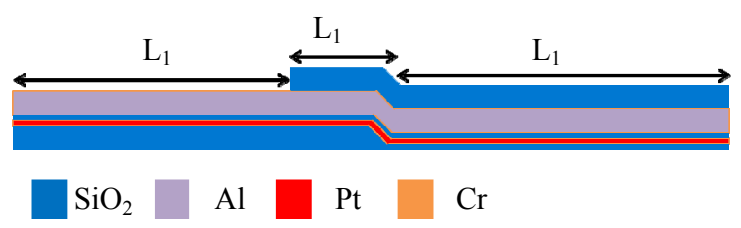

Figure 3: Cross section of S-shaped bimorph with all layers.

\section{Actuator Optimization}

Optimization of this design begins with addressing the need to reduce tilt. To address this, the following equation shows angular responsivity $\gamma$ in a single bimorph, which can be utilized as an approximate guideline for design constraints [11].

$$
\gamma=\frac{\theta_{T}}{\Delta T}=L_{b}\left(\alpha_{1}-\alpha_{2}\right) \beta_{r}
$$

In Eq. (4) the variables are as follows: thermal response angle from Joule heating $\theta_{\mathrm{T}}$; average temperate rise as a result of Joule heating $\Delta \mathrm{T}$; curvature coefficient of the bimorph combination $\beta_{\mathrm{r}}$; coefficient of thermal expansion from the top layer $\alpha_{1}$ and $\alpha_{2}$; and bimorph length $\mathrm{L}_{\mathrm{b}}$. From this equation it is found that the angular responsivity is directly proportional to the length of the bimorph in question. Reducing the bimorph lengths for each section by approximately $60 \%$ over the previous designs allows a significant and proportional reduction in angular responsivity, which strongly reduces sensitivity to signal nonlinearities and allows piston motion, when properly driven, to be the pure system output. This equation offers a guideline that does not consider the complex interactions between bimorph segments and compensated sections but presents a simple rule of thumb that may be validated most directly and clearly through experimental results.

In addition to angular responsivity, actuator stiffness is a critical aspect of mirror design as it informs robustness. It has been demonstrated that the compensating section of the actuator structure increases robustness of the actuator [8]. From this understanding, the overlap has been increased as a proportion of the S-shaped structure. This has yielded an approximately $30 \%$ increase in overlap to strengthen the actuator structure, which leads to an increase in device rigidity and vertical stiffness, all facets of a robust micromirror.

These improvements lower susceptibility of the mirror to rotation or breakage, which yields significant benefit for an intentionally mobile, piston actuating mirror. The tradeoff of such gains is requisite reduction in multimorph length, and in turn reduced actuation range. This has been handily addressed by stacking the FDSB actuators as illustrated in Fig. 4. This renders a mirror design with three stacked pairs of ladder actuators evenly spaced on a mirror edge and symmetric with the opposing edge (Fig. 5). The full mirror design is simulated via FEM simulation in COMSOL and shows the results of the optimizations of reducing angular responsivity and increasing rotational actuator stiffness [9].

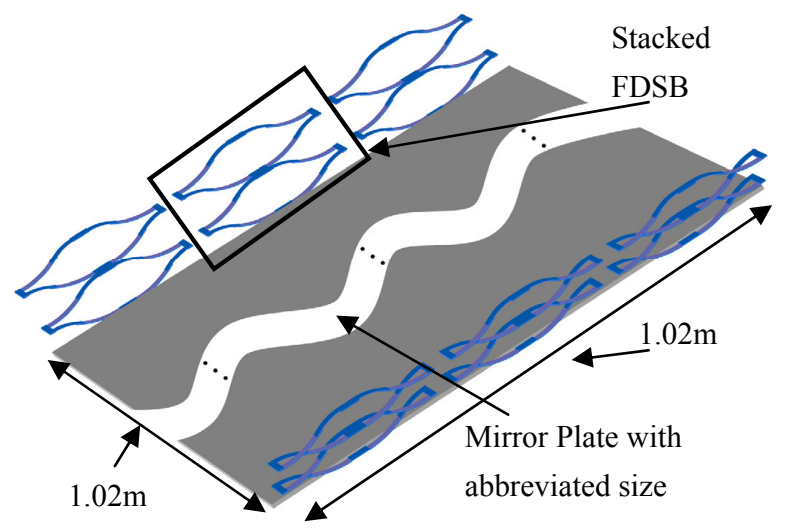

Figure 4: Full FEM of ladder actuator device with partially omitted mirror plate size.

\section{FABRICATION AND CHARACTERIZATION Device fabrication}

The fabrication process (Fig. 5) is a combined surface and bulk micromachining process that follows generally, with some variations, the process previously reported in [12]. The process utilizes silicon on insulator (SOI) wafer with the following features: a $40 \mu \mathrm{m}$-thick device layer, a $2 \mu \mathrm{m}$ buried oxide (BOX) layer and a $400 \mu \mathrm{m}$ handling layer. To begin, $1 \mu \mathrm{m}$ of PECVD $\mathrm{SiO}_{2}$ is deposited and then patterned through a buffered oxide etch primarily for the actuator (Fig. 5(a)). A $0.05 \mu \mathrm{m}$ PECVD $\mathrm{SiO}_{2}$ adhesion layer is then added to the wafer. Platinum for Joule heating is then sputtered and lifted off (Fig. 5(b)). PECVD $\mathrm{SiO}_{2}$ is again deposited for $0.2 \mu \mathrm{m}$ and patterned via dry etch to function as thermal isolation (Fig. 5(c)). Aluminum is evaporated for $1.0 \mu \mathrm{m}$ and lifted off for the actuator as well as traces (Fig. 5(d)). The final $\mathrm{SiO}_{2}$ layer is deposited via PECVD for $1.4 \mu \mathrm{m}$ and patterned via RIE dry etch primarily to complete the actuator (Fig. 5(e)). Next, photoresist is spun on a carrier wafer and applied to the frontside of the process wafer and oven-baked (Fig. 5(f)). After, backside bulk silicon anisotropic etch is performed until reaching the BOX. Thereafter, RIE etch removes the BOX (Fig. 5(g)). The carrier wafer is separated from the process wafer and the devices 
are split apart as chunks of devices aided by the previous backside anisotropic bulk silicon etch (Fig. 5(h)). Finally, the individual devices are released through a combination of anisotropic and isotropic etching in DRIE to release the devices and generate a final device configuration (Fig. 5(i)).

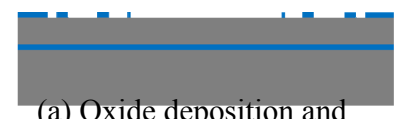

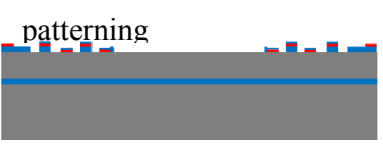

(c) Oxide deposition and contact opening

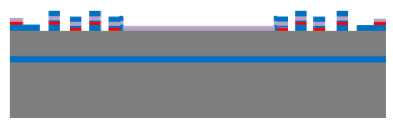

(e) Oxide deposition and

patterning

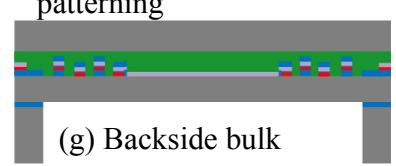

silicon and BOX etch

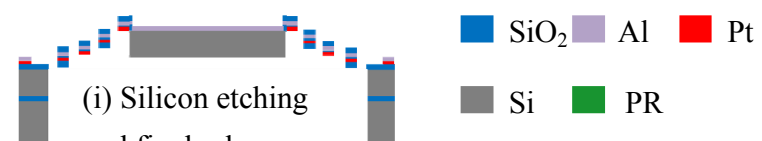

and final release

Figure 5: Fabrication process for device.

\section{Device characterization}

The final state of the device after fabrication and release is seen in Fig. 6(a). The mirror plate in Fig. 6(a) consists of the $40 \mu \mathrm{m}$-thick silicon device layer for the purposes of optical flatness. It can be seen in Fig. 6(c) that some sections of the bimorphs are exposed with $\mathrm{SiO}_{2}$ and the rest with $\mathrm{Al}$ while the embedded platinum for Joule heating is buried. The footprint of the device is $1.9 \mathrm{~mm} \times 1.9 \mathrm{~mm}$, and the mirror plate is $1.02 \mathrm{~mm} \times 1.02 \mathrm{~mm}$ which yields a fill factor of $28 \%$. From this design the individual ladder actuators have an average resistance of $15 \mathrm{ohms}$ with each individual ladder actuator capable of being driven through a distinct driving signal. The variance of these resistors is $+7 \mathrm{ohms}$ and $-2 \mathrm{ohms}$. The overall reduction of inactuator resistance is only $20 \%$. However, when factoring in the resistance from electrical paths over previous designs, the trace length is reduced by $\sim 70 \%$ [12]. Considering these two reductions, resistance, is reduced from $120 \mathrm{ohms}$ to $\sim 25 \mathrm{ohms}$. The remaining difference from this can be accounted for in fabrication variation.

The dc response of the device is measured by an Olympus BX51 microscope and a Quadra-Chek 200 geometry measuring system. From this setup, the mirror can step in piston motion through $90 \mu \mathrm{m}$ with a signal ranging from 0 to $1.2 \mathrm{~V}$ dc (Fig. 7). The observed linearity results from the reduced angular responsivity and the strong mechanical coupling of the ladder actuators. The maximum current draw of the complete device is $242 \mathrm{~mA}$. In addition to the displacement measurements, the tilt through the entire dc piston actuation range is measured. The maximum recorded tilt angle is $0.25^{\circ}$ during the entire dc piston actuation range on the actuator axis (Fig. 7). Additionally, there was no measureable off actuator tilt with this experimental setup.
This results in a tilt reduction of $85 \%$ over the previous electrothermal piston, low tilt micromirrors used in FTS applications [7].

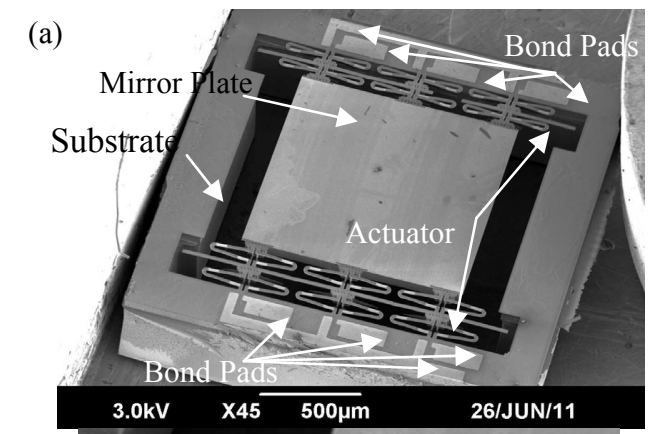

(b)

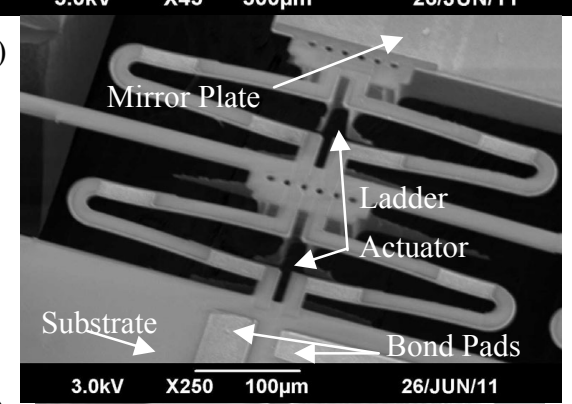

(c)

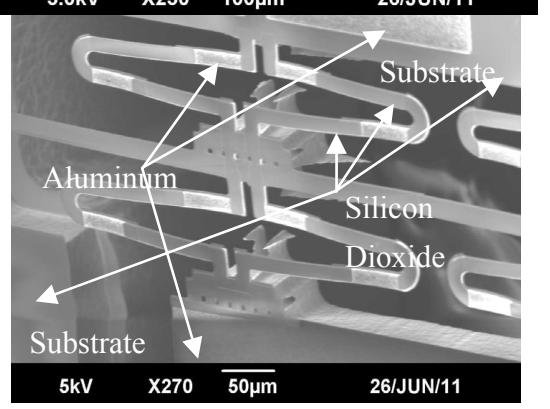

Figure 6: (a) SEM of the whole micromirror device, (b) SEM of the two-level ladder actuator, (c) SEM of the two-level ladder actuator at a different imaging angle with materials labeled (Platinum embedded and not shown).

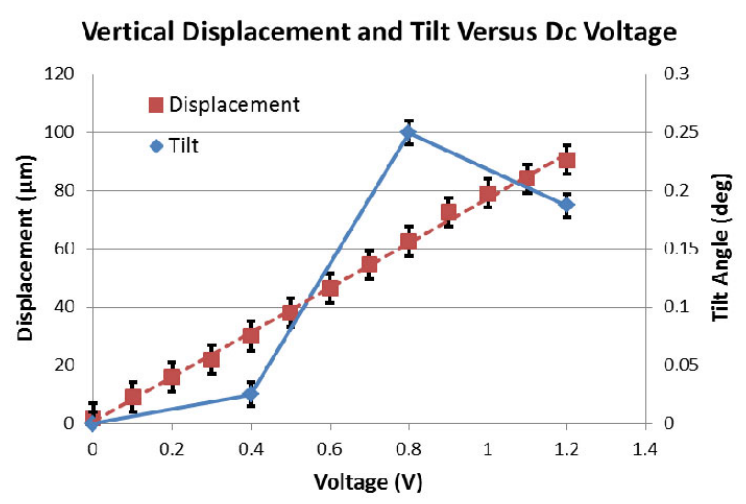

Figure 7: Recorded data points for displacement vs. given voltage at increments of $0.1 \mathrm{Vdc}$ and maximum tilt variation of only $0.25^{\circ}$.

For ac measurements, the micromirror is mounted on an optical breadboard for precise spacing from a 2D position sensitive detector (PSD). The driving signal ranges from 0.7 to $1.1 \mathrm{~V}$ at 
$0.33 \mathrm{~Hz}$. The signal is un-tuned and so does not adjust for layer thickness mismatch in the actuators from fabrication or for release step undercut of the sidewall and mirror edges. For a practical, unoptimized ac driving signal, the mirror produces a maximum of $0.22^{\circ}$ tilt on the actuator axis and $0.097^{\circ}$ on the off-actuator axis as measured via the PSD (Fig. 8).

Frequency characterization for the device also shows that the first resonant frequency or piston mode is found at $438 \mathrm{~Hz}$ with the second or torsional mode at $702 \mathrm{~Hz}$. This indicates that the design constraints have surpassed the frequency response over previous designs where $406 \mathrm{~Hz}$ was the torsional mode peak [12]. The results indicate a bandwidth amply wide to surpass the baseline definition of rapid-scan interferometers wherein the optical velocity is greater than $0.1 \mathrm{~cm}^{-1}[1]$.

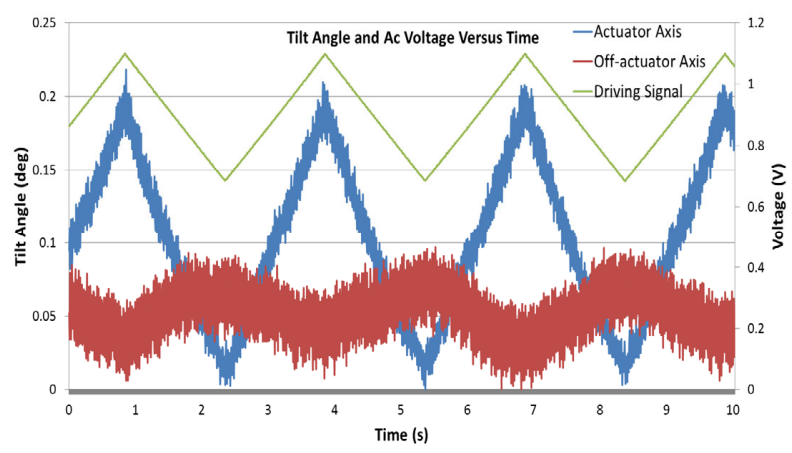

Figure 8: Tilt angle and recorded ac voltage applied to all actuators for piston motion yields a maximum tilt variation of only $0.22^{\circ}$ on the actuator axis and $0.097^{\circ}$ on the off-actuator axis.

\section{Characterization Results Interpretation}

The tilt of the mirror can be reduced in the ac and dc cases by tuning the driving voltage amplitudes to match the resistor variance of the actuators; however, due to resistor drift of several ohms during the heating and cooling cycles, such a static compensation is valid for only short intervals and requires regulation through feedback to remain beneficial.

As the tilt in the system ranges from minimum tilt to maximum tilt, it will be possible to utilize previously developed techniques of waveform shaping to correctly adjust the shift in mirror tilt while taking into account the thermal response time of the electrothermal micromirror [13]. The feedback component providing the information from this method will employ a heliumneon laser that will provide tilt information and real-time position information in accordance with established methods for reference sources [1][14]. This will permit effective integration into a practical system that will fulfill the requirements of a rapid-scan FTS system.

\section{CONCLUSION}

A unique ladder actuator that can provide ultralow tilt with large piston motion has been demonstrated. A micromirror based on such ladder actuators shows $25^{\circ}$ tilt at $90 \mu \mathrm{m}$ piston displacement. The maximum tilt is reduced by $85 \%$ compared to the previous design. This micromirror can produce a maximum wavenumber resolution of $55.6 \mathrm{~cm}^{-1}$. Prior large displacement MEMS mirrors have sacrificed stability in pure vertical displacement for larger range. In the pursuit of miniaturization, eliminating the need for physical tilt compensation streamlines and explains a practical path to a completely miniaturized system with concrete uses.

\section{ACKNOWLEDGEMENTS}

This work is supported by the National Science Foundation under the award \#0901711.

\section{REFERENCES}

[1] P. R. Griffiths and J.A. de Haseth, Fourier Transform Infrared Spectrometry, 2nd ed. Hoboken, N.J.: WileyInterscience, 2007.

[2] H. Xie and Z. M. Qi, "MEMS-Based Optical Chemical Sensors" in Semiconductor Device-Based Sensors for Gas, Chemical, and Biomedical Applications, F. Ren, Ed., CRC Press, 2011, ch. 7, pp. 267-302.

[3] K. Yu, D. Lee, U. Krishnamoorthy, N. Park, and O. Solgaard, "Micromachined Fourier transform spectrometer on silicon optical bench platform," Sensors and Actuators A: Physical, vol. 130-131, no. 0, pp. 523-530, Aug. 2006.

[4] O. Manzardo, H. P. Herzig, C. R. Marxer, and N. F. de Rooij, "Miniaturized time-scanning Fourier transform spectrometer based on silicon technology," Optics Letters, vol. 24, no. 23, pp. 1705-1707, Dec. 1999.

[5] T. Sandner, A. Kenda, C. Drabe, H. Schenk, and W. Scherf, "Miniaturized FTIR-spectrometer based on optical MEMS translatory actuator," Proceedings MOEMS and Miniaturized Systems VI, vol. 6466, pp. 646602-64660212, Jan. 2007.

[6] C. Solf, J. Mohr, and U. Wallrabe, "Miniaturized LIGA Fourier transformation spectrometer," Proceedings IEEE Sensors, 2003, vol. 2, pp. 773- 776, 22-24 Oct. 2003.

[7] L. Wu, A. Pais, S. R. Samuelson, S. Guo, and H. Xie, “A mirror-tilt-insensitive Fourier transform spectrometer based on a large vertical displacement micromirror with dual reflective surface," TRANSDUCERS 2009, pp. 2090-2093, 21-25 June 2009.

[8] K. Jia, S. Pal, and H. Xie, "An Electrothermal Tip-TiltPiston Micromirror Based on Folded Dual S-Shaped Bimorphs," Journal of Microelectromechanical Systems, vol. 18, no. 5, pp. 1004-1015, Oct. 2009.

[9] COMSOL, COMSOL Multiphysics 4.2, 2011.

[10] M. S. Weinberg, "Working equations for piezoelectric actuators and sensors," Journal of Microelectromechanical Systems, vol. 8, no. 4, pp. 529-533, Dec. 1999.

[11] H. Xie, S. Todd, A. Jain, and G. K. Fedder, "Single-Crystal Silicon Based Electrothermal MEMS Mirrors for Biomedical Imaging Applications" in MEMS/NEMS Handbook Volume 5: Medical Applications and MOEMS, C.T. Leondes, Ed., New York, N.Y.: Springer US, 2006, pp. 101-143.

[12] L. Wu, S. R. Samuelson, J. Sun, W. Lau, S. Choe, B. S. Sorg, K. Jia, and H. Xie, "A 2.8-MM imaging probe based on a high-fill-factor MEMS mirror and wire-bonding-free packaging for endoscopic optical coherence tomography," IEEE 24th International Conference on Micro Electro Mechanical Systems (MEMS), Cancun, Mexico, 2011, pp. 33-36.

[13] S. Pal and Huikai Xie, "Pre-Shaped Open Loop Drive of Electrothermal Micromirror by Continuous and Pulse Width Modulated Waveforms," IEEE Journal of Quantum Electronics, vol. 46, no. 9, pp. 1254-1260, Sep. 2010.

[14] R. J. Bell, Introductory Fourier Transform Spectroscopy. New York, N.Y.: Academic Press, 1972.

\section{CONTACT}

*S.R. Samuelson, tel: +1-352-846-0441; cap315@ufl.edu 